\title{
The Continuing Impacts of the COVID-19 \\ Pandemic in the United States: Findings from the 2021 Guttmacher Survey of Reproductive Health Experiences
}

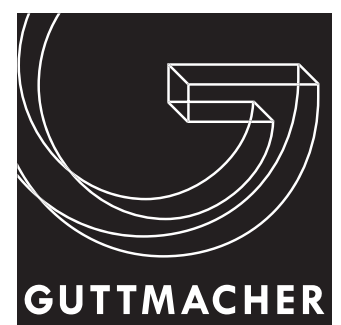

Laura D. Lindberg, Jennifer Mueller, Marielle Kirstein and Alicia VandeVusse

\section{KEY POINTS}

$\rightarrow$ The pandemic has continued to shift fertility preferences and impede access to sexual and reproductive health care, including contraceptive services.

$\rightarrow$ The impacts reported in the summer of 2021 are smaller than those reported earlier in the pandemic but remain pervasive.

$\rightarrow$ The pandemic continues to have disproportionate effects on the sexual and reproductive health of those already experiencing systemic social and health inequities.

$\rightarrow$ Telehealth services are bridging gaps in sexual and reproductive health care resulting from pandemic-related upheaval, particularly for those who already experience barriers to accessing health care.
Table of Contents 2

Findings 2

Discussion 8

Methodology Appendix 10

References 12 


\section{Introduction}

The COVID-19 pandemic is a public health crisis with major ramifications for people's personal lives, including their sexual and reproductive health. In early May 2020, we surveyed a national sample of cisgender women in the 2020 Guttmacher Survey of Reproductive Health Experiences (GSRHE). We found that just a few months into the COVID-19 pandemic, there were profound changes to respondents' sexual and reproductive health goals and behaviors and their access to care.' Other early studies validated these findings. ${ }^{2,3}$ Since May 2020, the course and impact of the pandemic have shifted, due to vaccination rates, new variants and relaxed restrictions. To provide an updated look at the ongoing impact of the pandemic on sexual and reproductive health, we fielded another national survey during July and August of 2021. The 2021 GSRHE had a substantially larger sample size than the previous one and was designed to include individuals with a broader range of gender identities. The expanded sample allows us to examine variations in several new, key areas, including gender identity, Asian and Pacific Islander racial identity, and the intersection of race and income.

In this report, we analyze the 2021 data, focusing on how respondents feel the COVID-19 pandemic has influenced their sexual and reproductive health in two core areas: fertility preferences and access to care, including use of telehealth. We note disparities according to individuals' race and ethnicity, sexual orientation, gender identity, income level and economic wellbeing. To assess the ongoing scope and magnitude of the impacts of the pandemic, we also examine findings on comparable measures from the 2020 and 2021 GSRHE studies.

These data provide four key findings:

- The pandemic has continued to shift fertility preferences and impede access to sexual and reproductive health care, including contraceptive services.
- The impacts reported in the summer of 2021 are smaller than those reported earlier in the pandemic but remain pervasive.

- The pandemic continues to have disproportionate effects on the sexual and reproductive health of those already experiencing systemic social and health inequities.

- Telehealth services are bridging gaps in sexual and reproductive health care resulting from pandemic-related upheaval, particularly for those who already experience barriers to accessing health care.

\section{Findings}

The 2021 GSRHE is a nationally representative, online survey administered by NORC between July 14, 2021 and August 9, 2021. (Figure 1 shows when the 2020 and 2021 surveys were conducted relative to the course of the pandemic.) We recruited respondents who were aged 18-49, had ever engaged in penile-vaginal sex and were assigned female at birth.* Respondents were recruited through a combination of a probability sample (NORC's AmeriSpeak ${ }^{\circledR}$ panel) and a nonprobability sample (online opt-in panel).
A total of 6,211 respondents completed the survey.

In this report, analyses of the 2021 GSRHE data are weighted for sampling selection, and demographic differences discussed in the text are statistically significant at the $p<.05$ level, unless otherwise noted. See additional information about the survey, sample and methods of analysis in the Methodology Appendix (page 10).

\section{The COVID-19 backdrop}

The 2021 GSRHE was conducted in the late summer, after COVID-19 vaccines had become widely available and as many economic, social and health activities had resumed after earlier shutdowns. Still, COVID-19 cases were rising differentially across the country, due to the uneven spread of the Delta variant and variable vaccination rates, among other things. We found that the pandemic continued to pose many health and financial challenges and to have a disproportionate impact on many of the respondents of color. ${ }^{\dagger}$

- One-quarter of respondents reported experiencing COVID-19 firsthand (24\%), through their own illness (11\%), the loss of loved ones to the disease (15\%) or both.

\section{FIGURE 1. Guttmacher Survey of Reproductive Health Experiences (GSRHE) fielding dates and U.S. COVID-19 cases}

No. of COVID-19 cases, March 2020-September 2021

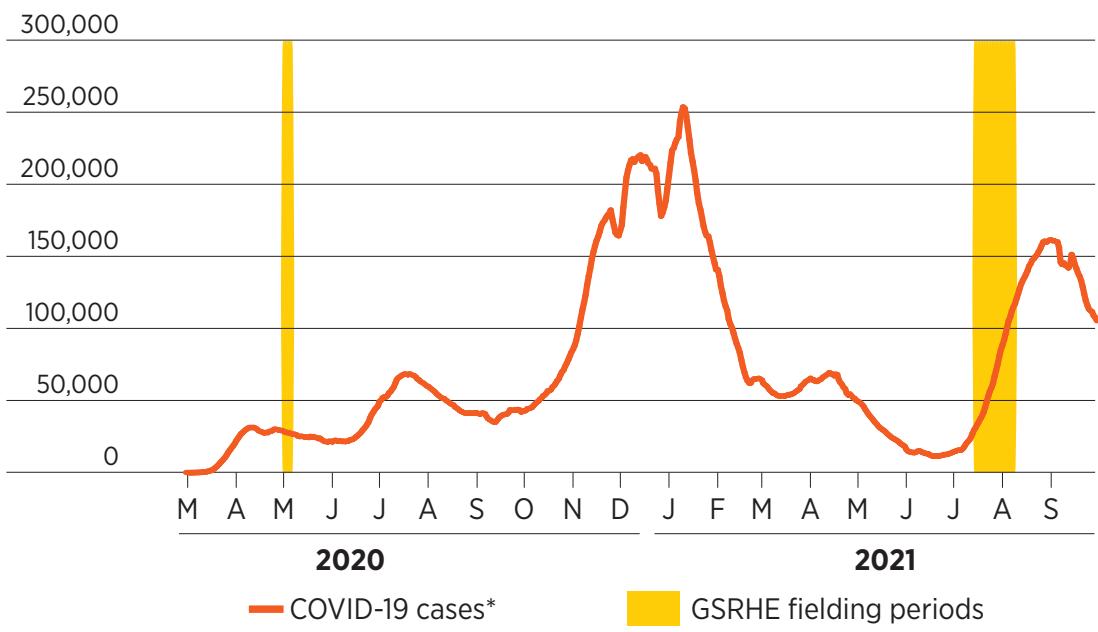

*Nearly all respondents (98\%) reported that they identified solely as women at the time of the survey.

*Seven-day moving average. Source: Centers for Disease Control and Prevention, COVID data tracker, October 2021, https://covid.cdc.gov/covid-data-tracker/\#trends_dailycases.

tThis group includes Hispanic, non-Hispanic Black, and non-Hispanic Asian and Pacific Islander respondents. 
- Hispanic and Black respondents were more likely to report these health outcomes than White respondents (33\% and $27 \%$, respectively, vs. $21 \%$ ). Asian and Pacific Islander respondents (22\%) did not differ from White respondents.

Financial instability and job losses impacted many 2021 respondents, as well.

- Overall, 39\% of respondents experienced financial instability or job loss. More than one-quarter of respondents (27\%) reported that they or someone in their household experienced a reduction in hours worked or lost a job because of COVID-19.

- Nearly one in four respondents (23\%) reported that in the last 12 months they fell behind on their rent or mortgage, had trouble meeting basic expenses or both.

- Hispanic and Black respondents were more likely to report any of these financial or employment challenges than White respondents ( $44 \%$ and $41 \%$, respectively, vs. $37 \%$ ). Asian and Pacific Islander respondents (31\%) did not differ from White respondents.

\section{Changes in fertility preferences}

The pandemic has transformed individuals' lives, including their preferences for when to have children and how many to have. For some people, the pandemic led to a desire to delay or reduce childbearing, while others reported a desire to increase or accelerate their childbearing plans. Fewer respondents reported any pandemicrelated change to their fertility preferences in 2021 than in 2020.

- In the 2021 GSRHE, more than one in five respondents (22\%) reported that because of the pandemic, they had changed their fertility plans in some way (Figure 2). Fifteen percent wanted fewer children or to have children later because of the pandemic, $11 \%$ reported that they wanted more children or to have children sooner because of the pandemic and $4 \%$ selected some combination of those options.

- By contrast, $41 \%$ of respondents in the 2020 GSRHE reported a change to their fertility plans, indicating that the impact of the pandemic on fertility preferences has abated for some.
Pandemic-related changes in fertility preferences have not occurred equally. Rather, respondents belonging to groups already experiencing systematic health and social inequities reported the greatest changes.

- Among respondents to the 2021 survey, pandemic-related shifts in fertility preferences were more likely to be experienced by respondents of color, LGB+ respondents, ${ }^{\ddagger}$ transgender and other genderdiverse respondents, ${ }^{\S}$ lower-income respondents, and those who experienced financial and employment difficulties in the past year than by their White, straight, cisgender, and financially better-off or employed counterparts (Figure 3, page 4).

- The fertility plans of Black and Hispanic respondents were disproportionately impacted regardless of their income. Higher-income Black (27\%) and Hispanic (26\%) respondents were more likely than lower-income White respondents (19\%) to report changes in their fertility plans.

\section{FIGURE 2. Fewer respondents in $\mathbf{2 0 2 1}$ than in $\mathbf{2 0 2 0}$ reported any pandemic-related changes to their fertility preferences or barriers to their sexual and reproductive health care.}

\section{Changed fertility preferences}

$100 \%$

80

60

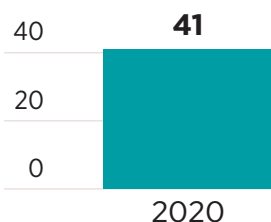

2020

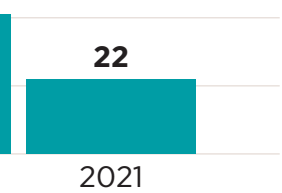

2021
Delayed or canceled contraceptive or other sexual and reproductive health care*

$100 \%$

80

60

40

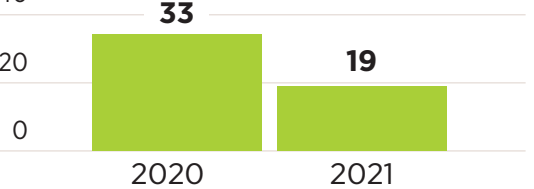

*In the past 12 months.
tRespondents were asked to report their sexual orientation with one or more of the following responses: straight, lesbian or gay, bisexual or pansexual, and other. We combined all responses other than straight into a single "LGB+" category.

§Respondents were asked to report their gender identity with one or more of the following responses: woman, man, nonbinary, transgender and other. 
FIGURE 3. Changes in fertility preferences in 2021 because of the COVID-19 pandemic were reported unequally by groups that experienced preexisting inequalities.

$\%$ of respondents reporting a change in the desired number or timing of children

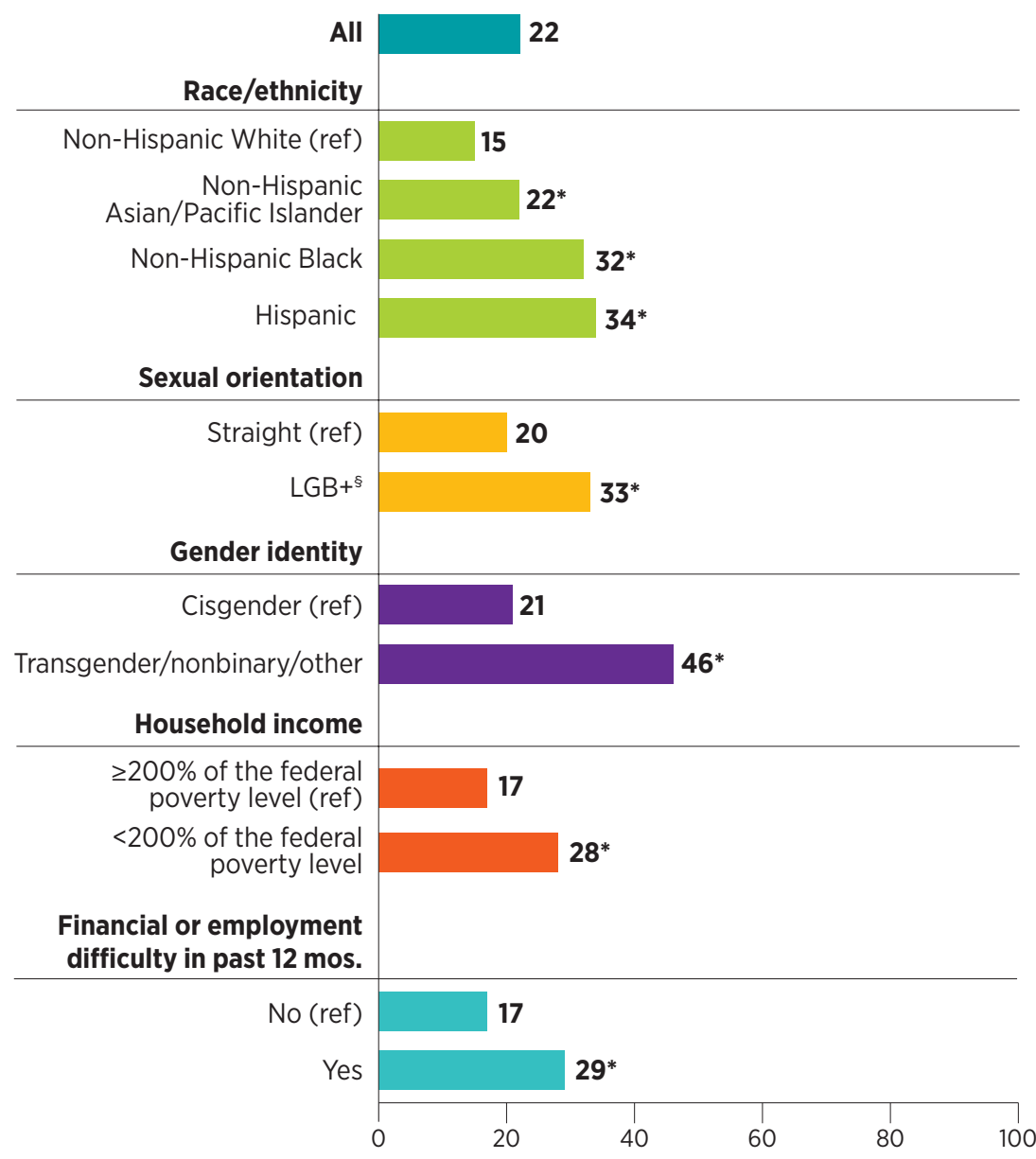

*Difference is statistically significant at $p<.05$. ${ }^{\circledR} \mathrm{LGB}+$ category includes responses of lesbian, gay, bisexual, pansexual and other. Note: ref=reference category. 
When asked why the pandemic caused changes to their childbearing plans, respondents offered a diverse set of reasons.

- Among respondents who reported that they wanted fewer children or to have children later because of the pandemic (15\%), the top reasons were "I'm concerned about money" (46\%; Figure 4), "It didn't seem like a good time to bring kids into the world" (46\%) or "I was scared to be pregnant or give birth during the pandemic" (40\%).

- Among those who reported that the pandemic inspired them to want more children or to have children sooner (11\%), the most commonly cited reason was that the pandemic "made me focus on what's important in life" (47\%). About one in three in this group (32\%) reported that their fertility plans changed because "I can work from home and/or have better worklife balance."
FIGURE 4. Respondents who reported pandemic-related changes to their fertility preferences in 2021 offered diverse reasons.

\begin{tabular}{|c|c|c|}
\hline Fertility change & Reasons reported & $\%$ \\
\hline \multirow{6}{*}{$\begin{array}{l}\text { Wanting to have } \\
\text { fewer children or to } \\
\text { have children later }\end{array}$} & I'm concerned about money & 46 \\
\hline & $\begin{array}{l}\text { It didn't seem like a good time to bring kids into } \\
\text { the world }\end{array}$ & 46 \\
\hline & $\begin{array}{l}\text { I was scared to be pregnant or give birth during } \\
\text { the pandemic }\end{array}$ & 40 \\
\hline & I'm concerned about work and my career & 34 \\
\hline & It made me reevaluate my priorities & 31 \\
\hline & I'm already caring for children or others & 19 \\
\hline \multirow{5}{*}{$\begin{array}{l}\text { Wanting to have } \\
\text { more children or } \\
\text { to have children } \\
\text { sooner }\end{array}$} & It made me focus on what's important in life & 47 \\
\hline & $\begin{array}{l}\text { I can work from home and/or have better work- } \\
\text { life balance }\end{array}$ & 32 \\
\hline & I have more free time/I'm working less & 23 \\
\hline & I'm in a better financial position & 23 \\
\hline & Now felt like as good a time as any & 23 \\
\hline
\end{tabular}

Note: Respondents were allowed to select more than one reason. 


\section{Access to contraception and other sexual and reproductive health services}

The pandemic created logistic and economic barriers to accessing contraceptive services and other sexual and reproductive health care that have continued over time. Still, fewer respondents in 2021 than in 2020 reported that their access to these kinds of care was negatively impacted by the pandemic. This may reflect the expansion of telehealth and other service delivery innovations that providers implemented in response to the challenges of the pandemic.

- In 2021, one in five respondents (19\%) reported that because of the pandemic, they had had to delay or cancel visiting a health care provider for sexual and reproductive health care or experienced trouble getting their contraceptive method in the 12 months prior to the survey (Figure 2 , page 3).

- Thirty-three percent of respondents reported these issues in 2020 (a period of more severe shutdowns and heightened uncertainty), and the change suggests that access to services has improved as the pandemic has progressed.

The 2021 survey documents sharp disparities by race/ethnicity, sexual orientation, gender identity and income in respondents' ability to access sexual and reproductive health services because of the pandemic.

- Respondents of color, LGB+ respondents, transgender and other gender-diverse respondents, ${ }^{* *}$ lower-income respondents, and those who experienced financial and employment difficulties in the past year were more likely than others to experience COVID-19-related barriers to this care (Figure 5).

- For some respondents of color, the pandemic had an impact on their access to care, regardless of their income. Higherincome Hispanic (23\%) and Black (22\%) respondents were more likely than lower-income White respondents (15\%) to report difficulty obtaining sexual and reproductive health care in the 12 months prior to the survey.
FIGURE 5. Pandemic-related delays or cancelations of contraceptive or other sexual and reproductive health care varied by respondents' characteristics in 2021.

$\%$ of respondents reporting delays or cancelation of care

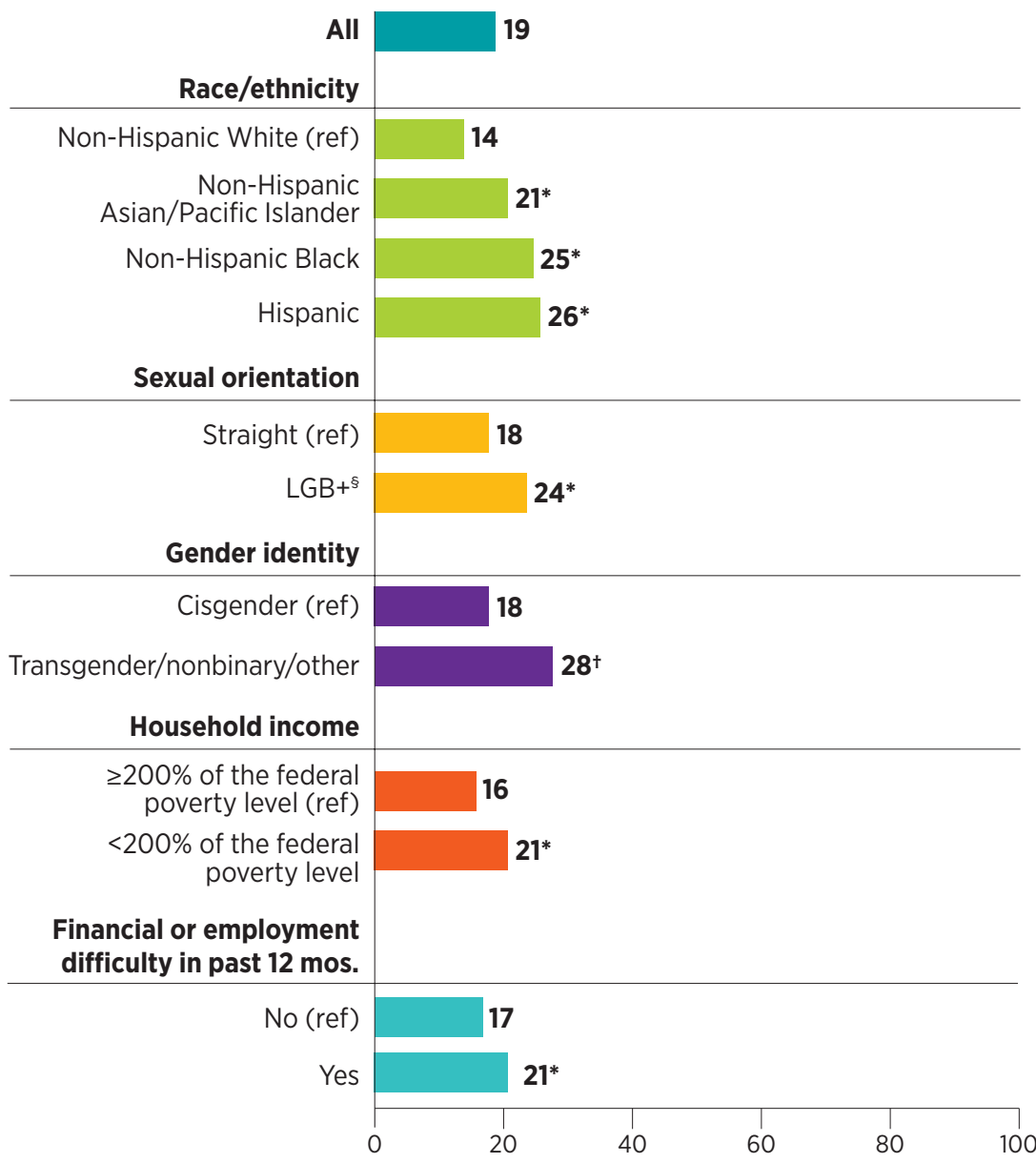

${ }^{*}$ Difference is statistically significant at $p<.05$. ${ }^{+}$Difference is statistically significant at $p<.07$. ${ }^{\S} \mathrm{LGB}+$ category includes responses of lesbian, gay, bisexual, pansexual and other. Note: ref=reference category.

${ }^{* *} p=.07$. 
Telehealth-i.e., video conferencing or telephone access to health care providers-and online contraception websites or apps, such as The Pill Club or GoodRx, are helping to bridge gaps in contraceptive care resulting from pandemic-related upheaval.

- Among those who received contraceptive care in the last six months, $17 \%$ used telehealth or online services for their most recent contraceptive care, $49 \%$ received care in-person and 34\% received care from a pharmacy, drugstore or some other place.

- Among those using telehealth or online services for contraceptive care, nearly half had not done so prior to the pandemic (44\%), suggesting that these services may be turned to when in-person care is less available.

- Use of telehealth or online services was more likely among respondents who reported experiencing pandemic-related delays or difficulties getting contraceptive care in the last six months than among those who did not experience issues seeking care (35\% vs. $15 \%)$.

- Groups who traditionally experience systemic barriers to accessing health care used telehealth or online services most commonly: respondents of color were more likely to use them than were White respondents (21-22\% vs. $13 \%$ ) and lowerincome respondents were more likely to use them than were higher-income respondents (20\% vs. 15\%; Figure 6).

- Respondents who used telehealth or online services for contraceptive care were less likely than those obtaining in-person care to rate the overall quality of care as excellent (47\% vs. 55\%).
FIGURE 6. Respondents of color and lower-income respondents used telehealth or an online app for contraceptive care more often than others in 2021.

$\%$ of respondents who received care online

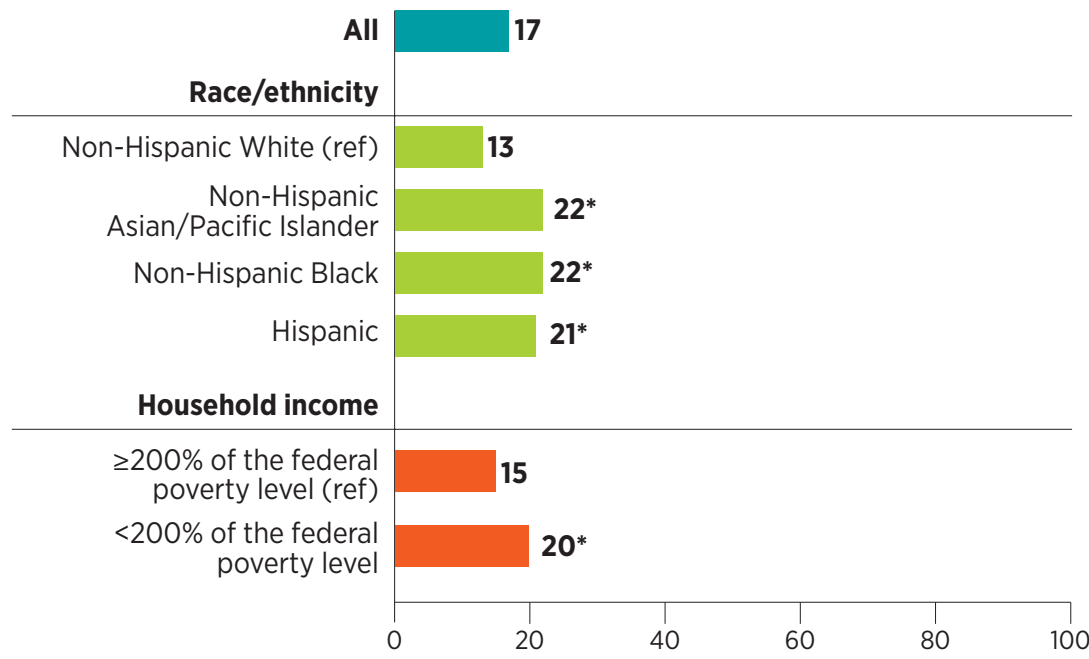

${ }^{*}$ Difference is statistically significant at $p<.05$. Note: ref=reference category. 


\section{Discussion}

The COVID-19 crisis and its unprecedented economic and social impacts have changed when people want to get pregnant, how many children they want, and if and how they are able to get the contraceptive services and other sexual and reproductive health care they need to make these fundamental life choices. But the impacts of the pandemic on these domains appear to be diminishing as the pandemic stretches on and COVID-19 transforms from an acute to a chronic threat. In the summer of 2021, when the second GSRHE was conducted, many areas of the country were attempting to "return to normal," despite rising COVID-19 cases in large swaths of the country. Against this backdrop, we find persistent, albeit less pervasive, impacts of the pandemic on fertility preferences and access to sexual and reproductive health care compared with findings from our 2020 survey. We also find a disproportionate effect on the sexual and reproductive health of respondents of color, LGB+ respondents, lower-income respondents, and those who experienced financial and employment difficulties in the past year. The larger sample size of this study allowed us to examine the intersecting impacts of race and income as well as the experiences of transgender and other gender-diverse respondents, documenting that they also were disproportionally impacted by the pandemic. Addressing these sexual and reproductive health inequities is central to achieving reproductive justice and ensuring that all people can decide when, whether and how to become parents, and have access to the support they need to take care of their families. ${ }^{4}$

\section{From "baby bust" to "fertility shock"}

Despite early conjecture that increased time at home would lead to a baby boom, in the early months of the pandemic, we found that many of our survey respondents wanted to delay pregnancy and have fewer children because of the pandemic. These changing fertility preferences have translated into declines in the birth rate in the United States ${ }^{5}$ and abroad. ${ }^{6}$ Many commentators noted the dramatic impact of school and child care closures on parents of young children-particularly mothers; this uneven care burden led to dramatically higher job loss among mothers than fathers during the early days of the COVID-19 pandemic.,8

However, as it has become clear that the pandemic's effects would be felt for years rather than weeks or months, fertility preferences may be stabilizing. Changes to fertility preferences were less prevalent in the summer of 2021 than in the spring of 2020. The length of the pandemic and the everchanging nature of its impact may have led some people to decide that prolonged delays or reductions in childbearing plans are unsustainable or unnecessary, leading COVID-19's impact to be less of a "baby bust" and more of a "fertility shock" with a severe initial drop but less pronounced long-term impacts. And, in both periods, some people responded to the pandemic's impacts by shifting their preferences toward accelerating or increasing their childbearing, although these changes were smaller than the shifts toward reduced or delayed fertility.

\section{Impacts of innovation in sexual and reproductive health care delivery}

With innovations and adaptations to the provision of sexual and reproductive health care, fewer respondents reported barriers to timely care in summer 2021 than they did in spring 2020. In the initial months of the pandemic, many cities and states instituted restrictions on so-called nonessential health care. ${ }^{9}$ Combined with shutdowns of schools, workplaces and other venues, many people may have avoided receiving sexual and reproductive health care in the wake of these dramatic disruptions. Furthermore, health care providers were stretched thin as they dealt with the need to divert resources to pandemic response.

Over time, in response to the logistical, financial and health challenges of providing care during the pandemic, providers experimented with innovative contraceptive method delivery options such as mail and curbside delivery, adapted their in-person care practices to reduce the risk of COVID19 exposure ${ }^{10,11}$ and expanded access to medication abortion. ${ }^{12}$ These pivots were supported by federal policy and state regulations that both loosened requirements for telehealth provision and expanded payment for telehealth services, facilitating a major expansion in the use of telehealth for contraceptive care. ${ }^{11,13}$

The impact of these shifts is evident in our findings-nearly one in five respondents who received recent contraceptive care accessed it via telehealth or online services. Many were new users of these methods of care, and many users reported difficulty accessing contraceptive care because of the pandemic, which may indicate that their use of telehealth was born of necessity rather than preference. It is concerning that those using telehealth for contraceptive care rated its quality lower than those receiving in-person care. Given this study's findings of greater use of telehealth by respondents of color and lower-income respondents, there is a need for further investigation to understand how telehealth use reflects personal preferences relative to systemic inequities in the availability, provision and quality of care. Moving forward, we must promote the development of equitable and high-quality telehealth care and not have it be a lesser standard and experience of care.

Even with increased uptake of telehealth and app-based care, gaps in sexual and reproductive health services remain, and COVID-19 continues to be named as a barrier to timely care by too many. Continued efforts are needed to broaden and support access to care, especially for those less well served by the health care system.

\section{Continued urgent need to address inequities}

Our new survey finds that the pandemic compounds existing social inequities by disproportionately affecting respondents of color, those who are LGB+, transgender and other gender-diverse respondents, lowerincome respondents and those experiencing financial and employment difficulties. The pandemic has been more disruptive in these respondents' lives than in the lives of their counterparts, resulting in higher COVIDrelated mortality, unemployment, school disruptions and other consequences. ${ }^{14-16}$

The results of these pandemic-related disruptions on sexual and reproductive health are illustrated by the heightened reported changes to disadvantaged respondents' fertility preferences and to their experience of barriers to timely sexual and reproductive 
health care. Preexisting inequities leave these respondents with fewer economic, social and institutional resources to shield against the impacts of the pandemic, fostering further inequities. Income alone did not cushion against these experiences; even when they had higher incomes, respondents of color were more likely than White respondents to have had their fertility preferences impacted by the pandemic and to have faced barriers to care. Our findings show the saliency of race/ethnicity in how the pandemic was experienced, suggesting that these inequities are rooted in structural racism.

The pandemic and its impact are not over, and people will continue to experience social and economic disruptions. Since the 2021 GSRHE was conducted, essential economic supports, including expanded unemployment insurance and the federal eviction moratorium, have ended, leaving people more vulnerable to economic instability. Policy responses to these challenges should prioritize communities that have borne the brunt of the pandemic and existing inequities. The national focus of this report does not illuminate state- and community-level variation in the impact of the pandemic, and differentiated government responses are likely needed going forward.

\section{Policy imperatives}

These national survey findings point to the need to strengthen health support systems and fight states' attacks on sexual and reproductive health writ large to ensure people can get the care they need during this pandemic and in the future. We need federal and state policies to support people economically and to reduce barriers to accessing health care so that individuals can select from a full range of fertility decisions, such as preventing or ending a pregnancy, having a healthy pregnancy and delivery, and parenting with dignity.

Pandemic or not, people should be able to achieve reproductive autonomy-they should have the resources and health care they need to control their own sexual and reproductive well-being. State and federal policymakers can take concrete steps to prioritize access to contraception, abortion and other essential sexual and reproductive health care. Guttmacher policy experts recommend they take the following steps:

- Focus on reducing inequities and center the needs of those most impacted and marginalized. Achieving reproductive health and justice requires adopting new policies that aim to address structural determinants of health inequities, such as the federal Black Maternal Health Momnibus Act that supports the health and well-being of all pregnant and parenting people, ${ }^{17}$ and the child tax credit $^{18}$ that offers means of support to those most impacted by the pandemic and ongoing inequities.

- Support public family planning clinics. The Biden-Harris administration has begun the process of restoring the nation's family planning program, Title $X$, after it sustained the double blows of the Trumpera "domestic gag rule" and the COVID-19 pandemic, both of which increased the challenges family planning clinics face in meeting the needs of patients. ${ }^{19,20}$ The Title $X$ network needs increased funding to ensure high-quality care is available to all who need it.

\section{- Support telehealth access and other service innovations to expand and ensure access to services. Policies should reflect that many reproductive health services can be safely and effectively provided via telehealth ${ }^{11,21}$ and should support people getting the care they need through the means they prefer, whether in person or via telehealth. Increased focus on quality of care and equity are needed to ensure that telehealth is a viable option for all patients.}

\section{- Ensure access to timely and affordable} abortion care. Key to protecting access to abortion is repealing the growing number of burdensome state and federal abortion restrictions and treating abortion as the essential care it is. ${ }^{22}$ With people wanting to postpone pregnancy or have fewer children and having difficulty accessing contraceptive care because of the pandemic, it has never been more important to meet the needs of those who want and need abortion care. 


\section{Methodology Appendix}

Survey recruitment and fielding for the 2021 Guttmacher Survey of Reproductive Health Experiences (GSRHE) was managed by NORC at the University of Chicago. Study participants were recruited over a four-week period between July 14, 2021 and August 9, 2021, via NORC's AmeriSpeak ${ }^{\circledR}$ panel, a probability-based panel designed to be representative of the U.S. household population, and Dynata's nonprobability online opt-in panel. The sample was selected from the AmeriSpeak ${ }^{\circledR}$ panel using sampling strata based on age, race/ethnicity, education and gender. For the nonprobability-based online sample, Dynata used enrollment targets for age, race/ethnicity and education. Dynata uses email invitations, phone alerts, banners and messaging on panel community sites to ensure inclusion of people with a diversity of motivations to take part in research. The final sample consisted of 6,211 complete responses, with 3,129 AmeriSpeak ${ }^{\circledR}$ respondents and 3,082 Dynata respondents. NORC calculated panel weights using their TrueNorth ${ }^{\circledR}$ calibration weighting service that allowed us to combine the completed AmeriSpeak ${ }^{\circledast}$ panel and the nonprobability online interviews. This combined sample approach has been used in prior studies of sexual and reproductive health, COVID-19 and other topics. ${ }^{23-25}$

Eligible respondents were those aged 18-49 who were assigned female at birth and who reported ever having had penile-vaginal sex. Participants who matched the eligibility criteria and agreed to participate in a research study were asked to complete an online survey that included a module on sexual and reproductive health and the COVID19 pandemic. Surveys were conducted in English and were Web-only.

Participants could skip any survey questions and could end the survey at any time. The survey collected no identifying information, and, to maintain respondents' confidentiality, NORC did not share respondents' identifying information with the study team. Panelists were offered a nominal incentive of about $\$ 3$ for completing the survey. Median completion time for the survey was 12 minutes. Study procedures were approved by the Guttmacher Institute and NORC institutional review boards.
NORC applied cleaning rules to the survey data for quality control by removing respondents who provided responses indicative of speeding through the survey, skipping survey questions, straight-lining responses to grid questions or answering open-ended questions with nonsensical answers; 474 respondents were removed for these reasons.

To check the generalizability of our sample, we compared the respondents in the 2021 GSRHE with a similarly defined sample in the 2017-2019 National Survey of Family Growth (NSFG) female dataset (Appendix Table 1). The 2021 GSRHE sample appears similar to that of the NSFG in terms of age, economic status, race/ethnicity, marital status and sexual orientation. However, the 2021 GSRHE sample is more highly educated and contains a lower proportion of respondents without children than the NSFG sample.

The addition of a nonprobability sample to the AmeriSpeak ${ }^{\circledR}$ panel provides the advantage of cost-effectively increasing sample size. This has permitted examination of experiences of subgroups often overlooked, such as respondents who identify as Asian or transgender. However, enrollment targets were used to ensure a more representative sample. The nonprobability sample may have other sampling and selection biases.

\section{APPENDIX TABLE 1.}

Percentage distribution of respondents aged 18-49 by demographic characteristics, 2017-2019 National Survey of Family Growth (NSFG) and 2021 Guttmacher Survey of Reproductive Health Experiences (GSRHE)

\begin{tabular}{|c|c|c|c|}
\hline \multicolumn{2}{|l|}{ Characteristic } & $\begin{array}{c}2017-2019 \text { NSFG } \\
(N=6,141) \\
\%\end{array}$ & $\begin{array}{c}2021 \text { GSRHE } \\
(\mathrm{N}=6,211) \\
\%\end{array}$ \\
\hline \multirow[t]{3}{*}{ Age } & $18-27$ & 17 & 16 \\
\hline & $28-38$ & 34 & 35 \\
\hline & $39-49$ & 49 & 49 \\
\hline \multirow{2}{*}{$\begin{array}{l}\text { Household } \\
\text { income }\end{array}$} & $\geq 200 \%$ of the federal poverty level & 57 & 57 \\
\hline & $<200 \%$ of the federal poverty level & 43 & 43 \\
\hline \multirow[t]{4}{*}{ Race/ethnicity } & Non-Hispanic White & 56 & 55 \\
\hline & Non-Hispanic Black & 14 & 14 \\
\hline & Hispanic & 20 & 21 \\
\hline & Non-Hispanic other/multiple races & 10 & 10 \\
\hline \multirow[t]{3}{*}{ Marital status } & Living with partner & 16 & 15 \\
\hline & Married & 45 & 47 \\
\hline & Other & 39 & 38 \\
\hline \multirow[t]{3}{*}{ Education } & High school graduate or less & 34 & 28 \\
\hline & Some college & 32 & 29 \\
\hline & College graduate or more & 34 & 43 \\
\hline \multirow{2}{*}{$\begin{array}{l}\text { Sexual } \\
\text { orientation }\end{array}$} & Straight & 86 & 85 \\
\hline & $\mathrm{LGB}^{*}$ & 14 & 15 \\
\hline \multirow{2}{*}{$\begin{array}{l}\text { No. of children } \\
\text { in household }\end{array}$} & None & 36 & 40 \\
\hline & One or more & 64 & 60 \\
\hline Total & & 100 & 100 \\
\hline
\end{tabular}

*For the NSFG, the LGB+ category includes responses of homosexual, gay, lesbian, bisexual and something else. For the GSRHE, it includes lesbian, gay, bisexual, pansexual and other. Source: Special tabulations of data from the 2017-2019 NSFG. 
An additional limitation of this study is that the 2021 GSRHE does not include adolescents younger than 18 , who have unique sexual and reproductive health needs and may have been differentially impacted by COVID-19 restrictions.

Data were analyzed with Stata 17.0 using the panel weights. We estimated bivariate logistic regression analyses for differences between subgroups in the $2021 \mathrm{GSRHE}$; all demographic differences presented in the text of this report were statistically significant at the $p<.05$ level, unless otherwise noted. Differences between point estimates in the 2020 and 2021 GSRHE are presented without formal statistical testing, given the differing sampling designs. 


\section{References}

1. Lindberg LD et al., Early Impacts of the COVID-19 Pandemic: Findings from the 2020 Guttmacher Survey of Reproductive Health Experiences, New York: Guttmacher Institute, 2020, doi:10.1363/2020.31482.

2. Diamond-Smith $\mathrm{N}$ et al., COVID-19's impact on contraception experiences: exacerbation of structural inequities in women's health, Contraception, 2021, S00107824(21)00369-3, doi:10.1016/j.contraception.2021.08.011.

3. Lin TK et al., The impact of the COVID-19 pandemic on economic security and pregnancy intentions among people at risk of pregnancy, Contraception, 2021, 103(6):380-385, doi:10.1016/j.contraception.2021.02.001.

4. Ross $L$ and Solinger R, Reproductive Justice: An Introduction, first ed., Berkeley, CA: University of California Press, 2017, https://www.ucpress.edu/ book/9780520288201/reproductive-justice.

5. Morse A, U.S. births declined during the pandemic, www.census.gov, Sept. 21, 2021, https://www.census.gov/library/ stories/2021/09/united-states-births-declined-during-the-pandemic.html.

6. Aassve A et al., Early assessment of the relationship between the COVID-19 pandemic and births in high-income countries, Proceedings of the National Academy of Sciences, 2021, 118(36), doi:10.1073/ pnas.2105709118.

7. Petts RJ, Carlson DL and Pepin JR, A gendered pandemic: childcare, homeschooling, and parents' employment during COVID-19, Gender, Work and Organization, 2020, 28(S2):515-534, doi:10.1111/ gwao.12614.

8. Heggeness ML et al., Tracking job losses for mothers of school-age children during a health crisis, www.census.gov, Mar. 3, 2021, https://www.census.gov/library/ stories/2021/03/moms-work-and-thepandemic.html.

9. Weigel G, Salganicoff A and Ranji U, Potential impacts of delaying "non-essential" reproductive health care, www.kff. org, June 24, 2020, https://www.kff.org/ womens-health-policy/issue-brief/potential-impacts-of-delaying-non-essentialreproductive-health-care/.

10. Tschann M et al., Family Planning Visits During the COVID-19 Pandemic: Phase 3
Results, Denver: Society of Family Planning, 2021, doi:10.46621/KNUB8934.

11. Weigel $\mathrm{G}$ et al., Opportunities and barriers for telemedicine in the U.S. during the COVID-19 emergency and beyond, $w W w$. kff.org, May 11, 2020, https://www.kff.org/ womens-health-policy/issue-brief/opportunities-and-barriers-for-telemedicine-in-theu-s-during-the-covid-19-emergency-andbeyond/.

12. Ramaswamy A et al., Medication abortion and telemedicine: innovations and barriers during the COVID-19 emergency, www.kff.org, June 16, 2021, https://www. kff.org/policy-watch/medication-abortiontelemedicine-innovations-and-barriersduring-the-covid-19-emergency/.

13. Medicaid emergency authority tracker: approved state actions to address COVID-19, wWw.kff.org, July 1, 2021, https://www.kff.org/coronavirus-covid-19/ issue-brief/medicaid-emergency-authoritytracker-approved-state-actions-to-addresscovid-19/.

14. Centers for Disease Control and Prevention, Disparities in deaths from COVID-19, 2020, https://www.cdc.gov/ coronavirus/2019-ncov/community/healthequity/racial-ethnic-disparities/disparitiesdeaths.html.

15. Broady K and Romer C, Despite June's positive jobs numbers, Black workers continue to face high unemployment, www.brookings.edu, July 2, 2021, https:// www.brookings.edu/blog/the-avenue/2021/07/02/despite-junes-positivejobs-numbers-black-workers-continue-toface-high-unemployment/.

16. Kuhfeld $M$ et al., Learning During COVID-19: Initial Findings on Students' Reading and Math Achievement and Growth, Portland, OR: NWEA, 2020, https:// www.nwea.org/research/publication/learning-during-covid-19-initial-findings-onstudents-reading-and-math-achievementand-growth/.

17. Keller LH et al., Reviving sexual and reproductive health and rights in the Biden-Harris era, Policy Analysis, New York: Guttmacher Institute, 2020, https://www. guttmacher.org/article/2020/11/revivingsexual-and-reproductive-health-andrights-biden-harris-era.

18. Parolin Z et al., Monthly poverty rates among children after the expansion of the
Child Tax Credit, Poverty and Social Policy Brief, New York: Columbia University, 2021, Vol. 5, No. 4.

19. Fowler $\mathrm{Cl}$, Gable J and Lasater B, Family Planning Annual Report: 2020 National Summary, Washington, DC: Office of Population Affairs, Office of the Assistant Secretary for Health, Department of Health and Human Services, 2020, https://opa.hhs. gov/research-evaluation/title-x-servicesresearch/family-planning-annual-report.

20. Dawson R, Trump administration's domestic gag rule has slashed the Title $X$ network's capacity by half, Policy Analysis, New York: Guttmacher Institute, 2020, https://www.guttmacher.org/ article/2020/02/trump-administrationsdomestic-gag-rule-has-slashed-title-xnetworks-capacity-half.

21. Keller LH and Dawson R, Family planning providers show creativity and resilience in response to the COVID-19 pandemic, Policy Analysis, New York: Guttmacher Institute, 2020, https://www.guttmacher. org/article/2020/06/family-planningproviders-show-creativity-and-resilienceresponse-covid-19-pandemic.

22. Nash E, For the first time ever, U.S. states enacted more than 100 abortion restrictions in a single year, Policy Analysis, New York: Guttmacher Institute, 2021, https://www.guttmacher.org/article/2021/10/first-time-ever-us-statesenacted-more-100-abortion-restrictionssingle-year.

23. Miller K et al., Question design during COVID-19, slide presentation for the National Center for Health Statistics webinar: Designing Survey Questions about COVID-19, Atlanta: Centers for Disease Control and Prevention Collaborating Center for Questionnaire Design and Evaluation Research, June 22, 2021, https:// www.cdc.gov/nchs/ccader/images/ Webinar-CCQDER-COVID-19.pdf.

24. Gupta RS et al., Prevalence and severity of food allergies among US adults, JAMA Network Open, 2019, 2(1):e185630, doi:10.1001/jamanetworkopen.2018.5630.

25. Schueler KE et al., Denial of tubal ligation in religious hospitals: consumer attitudes when insurance limits hospital choice, Contraception, 2021, 104(2):194201, doi:10.1016/j.contraception.2021.02.011. 


\section{Acknowledgments}

This report was written by Laura D. Lindberg, Jennifer Mueller, Marielle Kirstein and Alicia VandeVusse, all of the Guttmacher Institute. It was edited by Haley Ball and Lisa Melhado. The authors gratefully acknowledge the critical feedback and contributions to survey development from the following Guttmacher colleagues: Ruth Dawson, Joerg Dreweke, Madeleine Haas, Rachel Jones, Megan Kavanaugh and Kimberley Lufkin.

The data analyzed in this report were collected as part of the 2021 Guttmacher Survey of Reproductive Health Experiences, in collaboration with NORC at the University of Chicago. Funding for survey design and data collection was provided in part by the Eunice Kennedy Shriver National Institute of Child Health and Human Development of the National Institutes of Health (award numbers R01HD092396 and R01HD084473). All analyses presented here were conducted separately from this funding with generous support from The JPB Foundation. The content is solely the responsibility of the authors and does not necessarily represent the views of the National Institutes of Health or The JPB Foundation.

\section{Suggested citation:}

Lindberg LD et al., The Continuing Impacts of the COVID-19 Pandemic in the United States: Findings from the 2021 Guttmacher Survey of Reproductive Health Experiences, New York: Guttmacher Institute, 2021, https://www.guttmacher.org/report/continuing-impacts-covid19-pandemic-findings-2021-guttmacher-survey-reproductive-health.

doi: https://doi.org/10.1363/2021.33301

(c) Guttmacher Institute, 2021

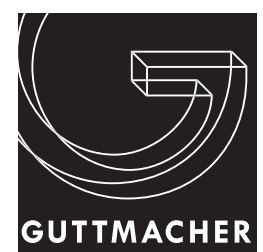

I N S T I T U T E

Good reproductive health policy starts with credible research

guttmacher.org

125 Maiden Lane

New York, NY 10038

212.248.1111

info@guttmacher.org 